\title{
Functional association between NUCKS1 gene and Parkinson disease: A potential susceptibility biomarker
}

\author{
Sarita Singh \& Prahlad Kishore Seth* \\ 4012091; Fax: 91-522-4012081; *Corresponding author \\ Received July 30, 2019; Accepted August 14, 2019; Published September 5, 2019
}

Biotech Park, Sector-G Jankipram, Kursi Road, Lucknow, India; Prahlad Kishore Seth - Email- sethpk.nasi@gmail.com; Phone: +91-522-

Abstract:

DOI: $10.6026 / 97320630015548$

Several Genome Wide Association Studies (GWASs) have reported that PARK16 gene locus possibly regulate the risk of Parkinson's disease (PD). It contains functionally interesting candidate genes for PD, regulated by number of SNPs. In present study rs823093 polymorphism in NUCKS1 gene has been evaluated as significant performer in PD though its mechanism is not yet known. Here various regulatory and functional analyses were performed using computational tools and information from databases. The rs823093 variant was predicted to locate in enhancer histone marks in blood and have strong transcription in various parts of brain, heart, kidney and liver. PhenoScanner (a database of human genotype-phenotype associations) identified significant associations of this variant with many other diseases and phenotypic conditions as well. Gene expression analysis shows significant association with multiple human tissues and multiple genes together with NUCKS1. Further, the post mortem brain samples showed diverse expressions of NUCKS1 gene in PD patients compared to healthy samples. Besides, the metabolite analysis shows significant association with serotonin a known neurotransmitter, and other 15 metabolites. In addition, NUCKS1 also showed co-expression with ZNF43 and PLIN1 genes involved in cell cycle regulation presume their association in PD. Thus, these data links NUCKS1 gene as a potential disease susceptibility biomarker for PD.

Keywords: NUCKS1; Parkinson; rs823093; GWAS; SNP

\section{Background:}

Parkinson's disease (PD) is the second most common neurodegenerative disorder after Alzheimer's disease and is reported to affect up to 1 million Americans over the age 55 and up to 10 million individuals worldwide [1]. In India, with an aging population and increased life expectancy, it is expected that the disease burden due to PD will be enormous, but there is no prospective study to estimate its incidence and mortality. The incidence rates (IRs) in different countries vary from 1.5 to 20 per 100,000 per year [2]. The disease is hallmarked by degeneration of a neurons specifically dopaminergic neurons between the substantianigra (SN) and the striatum. Investigators have reported that a significant number of dopamine producing cells are lost in the substantianigra of $\mathrm{PD}$ patients [3]. As these neurons are destroyed, the clinical signs which characterize PD such as the slowed movements, rigidity and tremors start to appear. Another key neuropathological mark of PD is the formation of Lewy bodies, which are cytoplasmic inclusions primarily, composed of the $\alpha$ synuclein protein. Lewy bodies have been reported in the dopaminergic neurons and other brain regions like the cortex and magnocellular basal forebrain nuclei [3]. 


\section{BIOINFORMATION \\ Discovery at the interface of physical and biological sciences}

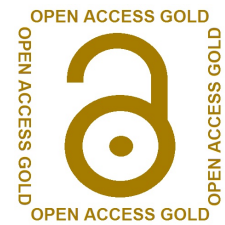

Besides the above-mentioned causes, interactions between genetic and environmental factors seem to play a critical role in the development of PD [4]. Several candidate genes and susceptibility loci causing monogenic familial forms of PD have been identified during number of genome wide association studies [5]. PARK16 locus, located on chromosome 1q32, having five candidate genes i.e. NUCKS1, RAB7L1, SLC41A1, SLA45A3 and PM20D1, is significantly associated with PD [6]. NUCKS1 gene encodes a nuclear protein, $27 \mathrm{kD}$ Nuclear casein kinase and cyclin-dependent kinase substrate 1 . The conserved regions of NUCKS1 contain several consensus phosphorylation sites for casein kinase II (CK2) and cyclin-dependent kinases (Cdk) and a basic DNA-binding domain. NUCKS1 is similar to the high mobility group (HMG) family, which dominates chromatin remodeling and regulates gene transcription [7]. NUCKS1 plays a significant role in various diseases as susceptibility or potential marker and involve in several regulatory mechanisms [8-12] Noticeably, NUCKS1 involves in cell growth and proliferation as well as in DNA repair [13]. Using genome wide Association Studies (GWAS) it was found that NUCKS1 is a susceptibility gene for many diseases and a single disease can be associated with multiple SNPs of NUCKS1. However, the same SNP of NUCKS1 for same disease when examined in different races showed that NUCKS1 has diverse expression. Besides, NUCKS1 genotypes exhibit distinct expression for certain diseases. Though exact roles of NUCKS1 in diseases remain unclear, a significant association of expression and transcription levels of NUCKS1 with PD has been observed [14, 15]. The rs823093 variant is located in the intron of NUCKS1 gene. The mechanism by which rs 823093 variant affects the PD pathogenesis is not yet known. In the present study, using bioinformatics approaches an attempt has been made to examine the functional association of rs823093 polymorphism and PD, with an aim to identify a susceptibility biomarker.

\section{Methodology:}

Regulatory and functional analysis

Regulatory and functional analysis of rs823093 variant was done using RegulomeDB database and HaploReg v4 tool, respectively. RegulomeDB could annotate genetic variants with known and predicted regulatory DNA elements which included regions of DNAase hypersensitivity, binding sites of transcription factors, promoter regions and binding motifs that play significant role in transcription regulation [16]. These datasets were collected from Gene Expression Omnibus (GEO), the Encyclopedia of DNA Elements (ENCODE) project, and published literature. HaploReg tool was used to annotate the non-coding variants which included information from the 1000 Genomes Project, chromatin state and protein binding annotation from the Roadmap Epigenomics and
ENCODE projects, sequence conservation across mammals, the effect of SNPs on regulatory motifs, and expression of genes [17, 18].

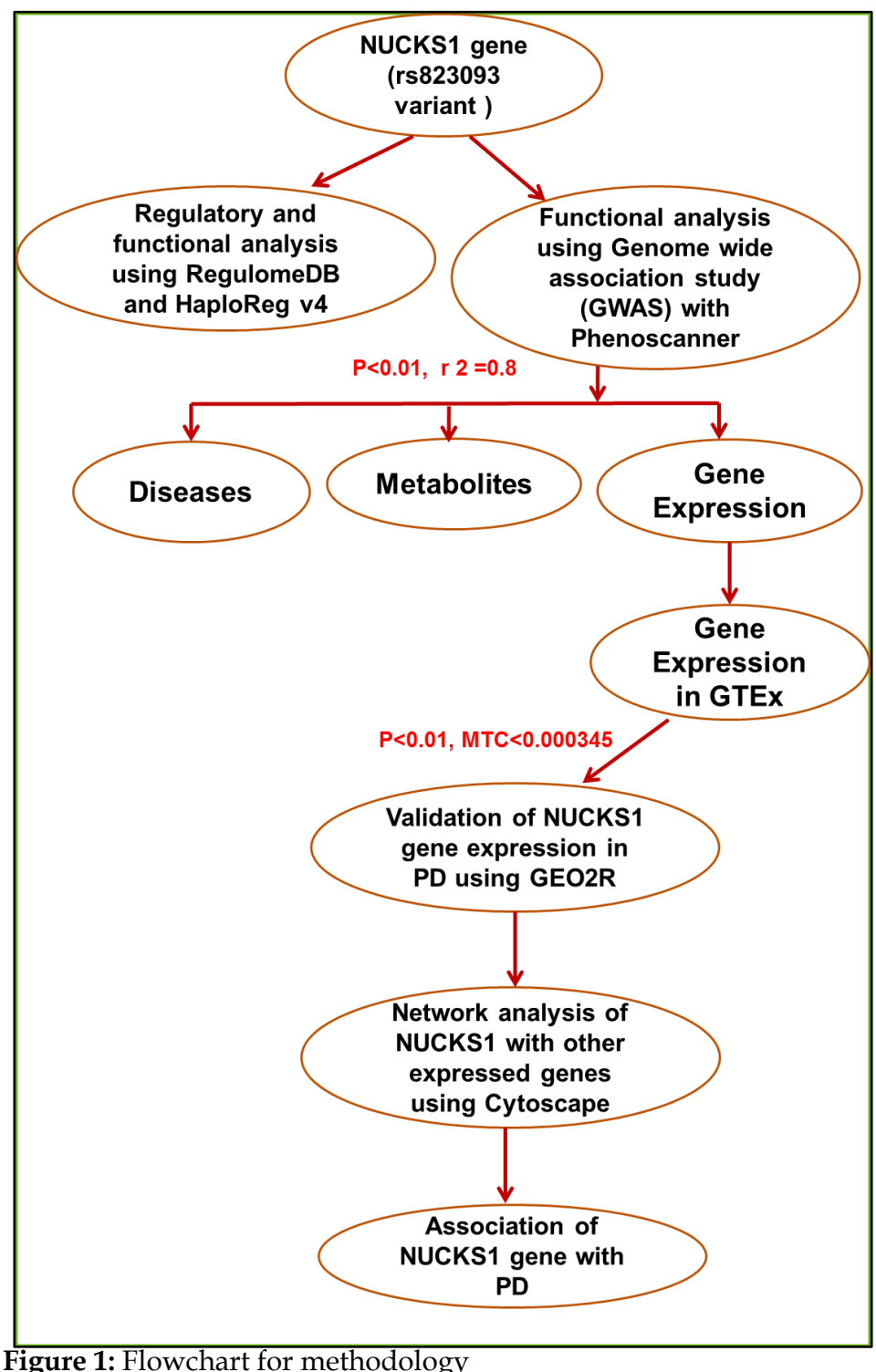

Figure 1: Flowchart for methodology 


\section{BIOINFORMATION \\ Discovery at the intertace of physical and hiological sciencess}

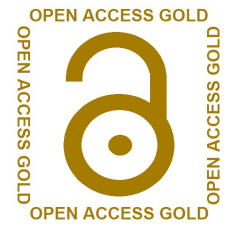

Functional analysis using Genome Wide Association Study (GWAS)

Functional analysis using Genome wide association study (GWAS) of rs823093 variant was done using PhenoScanner package. This package included publicly available large-scale GWAS data, about 3 billion associations and over 10 million unique single nucleotide polymorphisms (SNPs) and a comprehensive phenotypes data [19]. In the present study, three kinds of functional analyses including the GWAS of diseases, metabolites, and gene expression analysis were performed. The PhenoScanner included 88 GWAS datasets with 76 kinds of diseases or phenotypes to carry out a GWAS analysis [20]. To perform metabolites analysis, PhenoScanner consisted of two metabolomics datasets [21, 22]. For gene expression analysis, PhenoScanner included several datasets i.e. Geuvadis, GTEx (version 6), MuTHER, BIOSQTL, BLUEPRINT and Framingham etc.

Validation of NUCKS1 gene expression in Parkinson's disease Whole genome expression profiles in Parkinson's disease were analyzed to identify the responsible genes associated with Parkinson's disease. Here microarray expression data of total 25 samples (including 16 biopsy specimens of Parkinson's disease patients, and 9 healthy) from substantia nigra of postmortem human brain of Parkinson's disease patients was used [23]. A web tool GEO2R [24] to evaluate whether NUCKS1 gene is significantly deregulated in diseased cases compared with healthy samples at $\mathrm{P}$ $<0.01$ significance level was used. Additionally, GeneMANIA tool [25] in Cytoscape4.0 package was used to study the correlation between expressed genes associated with rs823093 variant.

\section{Chart for Methodology:}

Flowchart of complete methodology is shown in Figure 1.

\section{Results:}

Regulatory and functional analysis of rs823093 variant

Regulatory analysis of rs823093 variant shows score 6 in RegulomeDB, explaining that variant have binding motif i.e. OTX2 studies using Positional weight matrices (PWM) method. The histone modification study showed that the rs823093 variant is located in enhancer histone marks in blood and strong transcription in various parts of brain, heart, kidney and liver (Table 1 and Table S1). Functional analysis of rs823093 variant using HaploReg tool also shows enhancer histone marks in blood and six altered regulatory motifs i.e. DMBX1, FOXP1, LHX3, GSC, HMBOX1 and OBOX3. First three motifs are involves in brain related proteins.
Table 1: Histone modification analysis

\begin{tabular}{lll}
\hline Location & Chromatin State & Tissue \\
\hline chr1:205689200..205690000 & Genic enhancers & Blood \\
chr1:205684600..205691400 & Strong transcription & Fetal Brain Female \\
chr1:205685400..205690800 & Strong transcription & Fetal Brain Male \\
chr1:205686600..205690200 & Strong transcription & Brain Inferior Temporal \\
& & Lobe \\
chr1:205688000..205690600 & Strong transcription & Brain Substantia Nigra \\
chr1:205689000..205692200 & Strong transcription & Brain Hippocampus \\
chr1:205682800..205711200 & Strong transcription & Fiddle \\
chr1:205683400..205693800 & Strong transcription & Fetal Adrenal Gland \\
chr1:205684600..205693000 & Strong transcription & Fetal Kidney \\
chr1:205688000..205690000 & Strong transcription & Fetal Heart \\
chr1:205688000..205690200 & Strong transcription & Right Ventricle \\
chr1:205688000..205690400 & Strong transcription & Sigmoid Colon \\
chr1:205688400..205693200 & Strong transcription & Liver \\
chr1:205680400..205694200 & Quiescent/Low & Right Atrium \\
\hline
\end{tabular}

Association between rs823093 variant and type of disease or phenotype

GWAS of rs823093 variant identified thirty-eight significant associations at $P<0.01$. The variant is also significantly associated with other diseases or phenotypes besides Parkinson's disease such as Plateletcrit, Mucinous ovarian cancer, Chronic kidney disease, Particulate matter-associated QT prolongation, Monocyte percentage of white cells, Ulcerative colitis, Late onset Alzheimers disease, Neuroticism, Hip or knee osteoarthritis, Sporadic CreutzfeldtJakob disease and Inflammatory bowel disease etc. (details given in Table 2).

Association between rs823093 variant and gene expression The rs823093 variant shows fifty-three significant associations with gene expression at $\mathrm{P}<0.01$. The analysis predicted significant associations of rs823093 variant with gene expression in multiple human tissues like brain, pancreas, thyroid, cells transformed fibroblasts, colon sigmoid, heart left ventricle, liver, lung, skin, small intestine, stomach, and whole blood, as shown in Table 3. These expressed genes include PM20D1, RAB7L1, NUCKS1, SLC41A1, CFHR2, PLIN1, KRBA2, ZNF43, CBX1, PGA4, FGD1, NFASC, SERPINB11, SLC1A7, TMEM54, CCDC28A, SLC45A3 and MFSD4. Significantly, rs 823093 variant marks the expression of NUCKS1 gene in blood with $\mathrm{P}$ value $5.19 \mathrm{e}-09,3.62 \mathrm{e}-05 \& 3.41 \mathrm{e}-06$ and in brain frontal cortex 3.11e-04. 


\section{BIOINFORMATION \\ Discovery at the interface of physical and biological lsciencess}

Table 2: Association between rs823093 and type of disease or phenotype

\begin{tabular}{|c|c|c|c|}
\hline Disease or phenotype & PMID & P-value & $\begin{array}{l}\text { No. of } \\
\text { samples }\end{array}$ \\
\hline Prostate specific antigen levels & 25434496 & $5.00 \mathrm{e}-13$ & NA \\
\hline Parkinson's disease & 22438815 & $1.38 \mathrm{e}-11$ & 4258 \\
\hline Parkinson's disease & 19915576 & $4.88 \mathrm{e}-09$ & 3509 \\
\hline Parkinson's disease & 19915575 & $7.29 \mathrm{e}-08$ & 5691 \\
\hline Parkinson's disease & 21248740 & $7.29 \mathrm{e}-08$ & 2796 \\
\hline Parkinson's disease & 25064009 & $2.22 \mathrm{e}-06$ & 108990 \\
\hline Plateletcrit & 27863252 & $2.58 \mathrm{e}-04$ & 173480 \\
\hline Parkinson's disease & 21738487 & $1.90 \mathrm{e}-04$ & 33050 \\
\hline $\mathrm{HbA1c}$ & 28898252 & $1.19 \mathrm{e}-03$ & 123665 \\
\hline Body mass index & 28892062 & $9.80 \mathrm{e}-04$ & 173430 \\
\hline Body mass index in males greater than 50 years of age & 26426971 & $1.70 \mathrm{e}-03$ & 92442 \\
\hline Body mass index females & 28892062 & $3.95 \mathrm{e}-04$ & 82438 \\
\hline Mucinous ovarian cancer & 28346442 & $2.60 \mathrm{e}-03$ & 42090 \\
\hline Chronic kidney disease & 26831199 & $3.30 \mathrm{e}-03$ & 117165 \\
\hline Childhood BMI & 26604143 & $3.55 \mathrm{e}-03$ & 35669 \\
\hline Chronic kidney disease & 20383146 & $3.90 \mathrm{e}-03$ & 62237 \\
\hline Insulin sensitivity index adjusted for BMI interaction & 27416945 & $4.10 \mathrm{e}-03$ & 16753 \\
\hline Particulate matter-associated QT prolongation & 28749367 & $4.56 \mathrm{e}-03$ & 22158 \\
\hline Urea & 28887542 & $4.66 \mathrm{e}-03$ & 9961 \\
\hline ovarian cancer & 28346442 & $5.05 \mathrm{e}-03$ & 42895 \\
\hline Monocyte percentage of white cells & 27863252 & $5.24 \mathrm{e}-03$ & 173480 \\
\hline Ulcerative colitis & 26192919 & $5.30 \mathrm{e}-03$ & 27432 \\
\hline Body mass index adjusted for physical activity in males & 28448500 & $5.85 \mathrm{e}-03$ & 84503 \\
\hline Testosterone & 28887542 & $6.06 \mathrm{e}-03$ & 4387 \\
\hline Body mass index & 25673413 & $6.22 \mathrm{e}-03$ & 339224 \\
\hline Late onset Alzheimer's disease & 21390209 & $6.36 \mathrm{e}-03$ & 3595 \\
\hline Neuroticism & 27089181 & $6.44 \mathrm{e}-03$ & 170911 \\
\hline Hip or knee osteoarthritis & 22763110 & $6.74 \mathrm{e}-03$ & 18419 \\
\hline Waist circumference in female smokers & 28443625 & $7.42 \mathrm{e}-03$ & 20595 \\
\hline Body mass index in physically inactive individuals & 28448500 & $8.00 \mathrm{e}-03$ & 42066 \\
\hline Diabetic nephropathy & 16775037 & $8.01 \mathrm{e}-03$ & 1795 \\
\hline Body mass index adjusted for smoking in males & 28443625 & $8.38 \mathrm{e}-03$ & 102746 \\
\hline Body mass index in physically inactive individuals & 28448500 & $9.08 \mathrm{e}-03$ & 46393 \\
\hline Sporadic Creutzfeldt Jakob disease & 22210626 & $9.23 e-03$ & 7872 \\
\hline Inflammatory bowel disease & 26192919 & $9.42 \mathrm{e}-03$ & 34652 \\
\hline Granulocyte percentage of myeloid white cells & 27863252 & $9.58 \mathrm{e}-03$ & 173480 \\
\hline Body mass index in male non-smokers & 28443625 & $9.88 \mathrm{e}-03$ & 78101 \\
\hline Albumin & 28887542 & $9.93 e-03$ & 9961 \\
\hline
\end{tabular}

Table 3: Association between rs823093 and gene expression

\begin{tabular}{|c|c|c|c|c|c|c|c|}
\hline Gene & Tissue & No. of sample & Beta & SE & P-value & PMID & Source \\
\hline PM20D1 & Adipose subcutaneous & 385 & 0.4991 & 0.1489 & $9.02 \mathrm{e}-04$ & 25954001 & GTEx \\
\hline PM20D1 & Testis & 225 & 0.6166 & 0.1796 & $7.36 \mathrm{e}-04$ & 25954001 & GTEX \\
\hline PM20D1 & Whole blood & 2116 & NA & NA & $1.22 \mathrm{e}-30$ & 27918533 & BIOSQTL \\
\hline RAB7L1 & Adipose subcutaneous & 385 & -0.3531 & 0.09168 & $1.42 \mathrm{e}-04$ & 25954001 & GTEx \\
\hline RAB7L1 & Artery tibial & 388 & -0.362 & 0.08334 & $1.89 \mathrm{e}-05$ & 25954001 & GTEx \\
\hline RAB7L1 & Brain anterior cingulate cortex BA24 & 109 & -0.6235 & 0.1707 & $4.42 \mathrm{e}-04$ & 25954001 & GTEx \\
\hline RAB7L1 & Brain cortex & 136 & -0.4474 & 0.1162 & $1.96 \mathrm{e}-04$ & 25954001 & GTEx \\
\hline RAB7L1 & Breast mammary tissue & 251 & -0.3681 & 0.09399 & $1.23 \mathrm{e}-04$ & 25954001 & GTEx \\
\hline RAB7L1 & Esophagus mucosa & 358 & -0.2519 & 0.07573 & $9.94 \mathrm{e}-04$ & 25954001 & GTEx \\
\hline RAB7L1 & Esophagus muscularis & 335 & -0.3319 & 0.0768 & $2.14 \mathrm{e}-05$ & 25954001 & GTEx \\
\hline RAB7L1 & Heart left ventricle & 272 & -0.3227 & 0.08435 & $1.70 \mathrm{e}-04$ & 25954001 & GTEx \\
\hline RAB7L1 & Muscle skeletal & 491 & -0.346 & 0.06334 & $8.01 \mathrm{e}-08$ & 25954001 & GTEx \\
\hline RAB7L1 & Skin sun exposed lower leg & 414 & -0.3987 & 0.07704 & $3.86 \mathrm{e}-07$ & 25954001 & GTEx \\
\hline RAB7L1 & Thyroid & 399 & -0.3763 & 0.07258 & $3.77 \mathrm{e}-07$ & 25954001 & GTEx \\
\hline RAB7L1 & Monocytes & 194 & -0.9174 & 0.243 & $1.60 \mathrm{e}-04$ & 27863251 & BLUEPRINT \\
\hline RAB7L1 & Neutrophils & 192 & -0.8078 & 0.2442 & $9.42 \mathrm{e}-04$ & 27863251 & BLUEPRINT \\
\hline
\end{tabular}



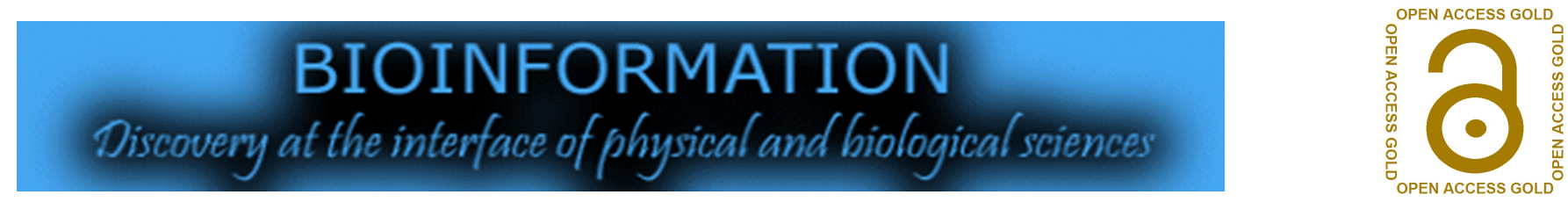

\begin{tabular}{|c|c|c|c|c|c|c|c|}
\hline RAB7L1 & Adipose visceral omentum & 313 & -0.3261 & 0.07763 & $3.65 \mathrm{e}-05$ & 25954001 & GTEx \\
\hline RAB7L1 & Artery aorta & 267 & -0.4965 & 0.1201 & $5.11 \mathrm{e}-05$ & 25954001 & GTEx \\
\hline RAB7L1 & Brain hippocampus & 111 & -0.9282 & 0.1895 & $4.29 \mathrm{e}-06$ & 25954001 & GTEx \\
\hline RAB7L1 & Colon sigmoid & 203 & -0.4021 & 0.1047 & $1.75 \mathrm{e}-04$ & 25954001 & GTEx \\
\hline RAB7L1 & Esophagus gastroesopha-geal junction & 213 & -0.3007 & 0.07885 & $1.89 \mathrm{e}-04$ & 25954001 & GTEx \\
\hline RAB7L1 & Heart atrial appendage & 264 & -0.3142 & 0.0907 & $6.44 \mathrm{e}-04$ & 25954001 & GTEx \\
\hline RAB7L1 & Nerve tibial & 361 & -0.2752 & 0.07424 & $2.50 \mathrm{e}-04$ & 25954001 & GTEx \\
\hline RAB7L1 & Pancreas & 220 & -0.4918 & 0.1111 & $1.63 \mathrm{e}-05$ & 25954001 & GTEx \\
\hline RAB7L1 & Brain hypothalamus & 108 & -0.9381 & 0.2511 & $3.35 \mathrm{e}-04$ & 25954001 & GTEx \\
\hline RAB7L1 & Stomach & 237 & -0.4187 & 0.1214 & $6.84 \mathrm{e}-04$ & 25954001 & GTEx \\
\hline RAB7L1 & Whole blood & 2116 & NA & NA & $1.35 \mathrm{e}-21$ & 27918533 & BIOSQTL \\
\hline RAB7L1 & Lung & 383 & -0.3165 & 0.08726 & $3.35 e-04$ & 25954001 & GTEx \\
\hline NUCKS1 & Brain frontal cortex BA9 & 118 & -0.5593 & 0.1495 & $3.11 \mathrm{e}-04$ & 25954001 & GTEx \\
\hline NUCKS1 & Prostate & 132 & 0.4927 & 0.1391 & $4.28 \mathrm{e}-04$ & 25954001 & GTEx \\
\hline NUCKS1 & Whole blood & 2116 & NA & NA & $5.19 \mathrm{e}-09$ & 27918533 & BIOSQTL \\
\hline NUCKS1 & Peripheral blood & 5311 & NA & NA & $3.41 \mathrm{e}-06$ & 24013639 & Westra-H \\
\hline NUCKS1 & Lung & 278 & 0.2174 & 0.06255 & $6.05 \mathrm{e}-04$ & 25954001 & GTEx \\
\hline NUCKS1 & Whole blood & 369 & 0.1994 & 0.04756 & $3.62 \mathrm{e}-05$ & 25954001 & GTEx \\
\hline SLC41A1 & Thyroid & 278 & -0.3251 & 0.09241 & $5.21 \mathrm{e}-04$ & 25954001 & GTEx \\
\hline SLC41A1 & Thyroid & 399 & -0.2909 & 0.06974 & $3.88 \mathrm{e}-05$ & 25954001 & GTEx \\
\hline SLC41A1 & Lymphoblasto-id cell lines & 462 & NA & NA & $3.53 \mathrm{e}-08$ & 24037378 & Geuvadis \\
\hline SLC41A1 & Brain anterior cingulate cortex BA24 & 109 & -0.467 & 0.1296 & $5.21 \mathrm{e}-04$ & 25954001 & GTEx \\
\hline CFHR2 & Peripheral blood monocytes & 1490 & NA & NA & $4.47 \mathrm{e}-06$ & 20502693 & Zeller \\
\hline PLIN1 & Whole blood & 5257 & -0.0209 & 0.00473 & $1.12 \mathrm{e}-05$ & 28122634 & Framingham \\
\hline KRBA2 & Whole blood & 5257 & NA & NA & $2.69 \mathrm{e}-05$ & 28122634 & Framingham \\
\hline ZNF43 & Whole blood & 5257 & 0.0435 & 0.01073 & $5.13 e-05$ & 28122634 & Framingham \\
\hline CBX1 & Whole blood & 5257 & 0.0352 & 0.00876 & $5.95 \mathrm{e}-05$ & 28122634 & Framingham \\
\hline PGA4 & Whole blood & 5257 & -0.042 & 0.01047 & $6.12 \mathrm{e}-05$ & 28122634 & Framingham \\
\hline FGD1 & Whole blood & 5257 & 0.013 & 0.00326 & $6.81 \mathrm{e}-05$ & 28122634 & Framingham \\
\hline NFASC & Prostate & 87 & 0.7023 & 0.1901 & $4.49 \mathrm{e}-04$ & 25954001 & GTEx \\
\hline NFASC & Pituitary & 87 & -0.5927 & 0.1395 & $6.98 \mathrm{e}-05$ & 25954001 & GTEx \\
\hline SERPINB11 & Whole blood & 5257 & -0.0322 & 0.00813 & $7.55 \mathrm{e}-05$ & 28122634 & Framingham \\
\hline SLC1A7 & Whole blood & 5257 & -0.0192 & 0.00485 & $7.56 \mathrm{e}-05$ & 28122634 & Framingham \\
\hline TMEM54 & Whole blood & 5257 & -0.0253 & 0.00644 & $8.55 e-05$ & 28122634 & Framingham \\
\hline CCDC28A & Whole blood & 5257 & 0.0227 & 0.00583 & $9.89 \mathrm{e}-05$ & 28122634 & Framingham \\
\hline SLC45A3 & Adipose subcutaneous & 385 & 0.2693 & 0.07622 & $4.72 \mathrm{e}-04$ & 25954001 & GTEx \\
\hline MFSD4 & Cells transformed fibroblasts & 272 & 0.585 & 0.1505 & $7.32 \mathrm{e}-04$ & 25954001 & GTEx \\
\hline
\end{tabular}

$\mathrm{SE}=$ Standard Error; Beta $=$ Regression coefficient based on effect allele i.e. effect allele regulates increased and decreased expression of nearby gene; P-value $<0.01$

Table 4: Association between rs823093 and metabolites

\begin{tabular}{llllll}
\hline Metabolite & No. of sample & Beta & SE & P-value & PMID \\
\hline Glycerol & 1735 & NA & NA & $1.60 \mathrm{e}-04$ & 21886157 \\
Isobutyrylcarnitine & 1725 & NA & NA & $3.10 \mathrm{e}-04$ & 21886157 \\
Pantothenate & 911 & NA & NA & $3.20 \mathrm{e}-04$ & 21886157 \\
Lactate & 24871 & 0.06257 & 0.02158 & $4.65 \mathrm{e}-03$ & 27005778 \\
4-acetamidobutanoate & 6523 & 0.016 & 0.0057 & $4.92 \mathrm{e}-03$ & 24816252 \\
LDL & 19273 & 0.05838 & 0.02064 & $4.99 \mathrm{e}-03$ & 27005778 \\
Serotonin (5HT) & 5791 & 0.0281 & 0.0106 & $5.04 \mathrm{e}-03$ & 24816252 \\
Gamma-glutamylleucine & 7354 & 0.0139 & 0.005 & $5.04 \mathrm{e}-03$ & 24816252 \\
Phosphate & 7341 & -0.0113 & 0.0042 & $7.04 \mathrm{e}-03$ & 24816252 \\
Cholesterol & 7365 & -0.0127 & 0.0048 & $7.41 \mathrm{e}-03$ & 24816252 \\
Caprylate & 7355 & -0.0154 & 0.0058 & $7.43 \mathrm{e}-03$ & 24816252 \\
Oleate & 7323 & -0.0178 & 0.0067 & $7.58 \mathrm{e}-03$ & 24816252 \\
Heptanoate & 7353 & -0.0295 & 0.0112 & $8.19 \mathrm{e}-03$ & 24816252 \\
7-methylguanine & 5804 & 0.0349 & 0.0133 & $8.45 \mathrm{e}-03$ & 24816252 \\
Glucose & 7325 & -0.0099 & 0.0038 & $9.16 \mathrm{e}-03$ & 24816252 \\
N-acetylornithine & 7146 & 0.0326 & 0.0126 & $9.49 \mathrm{e}-03$ & 24816252 \\
\hline
\end{tabular}

$\mathrm{SE}=$ Standard Error; Beta $=$ Regression coefficient based on effect allele i.e. effect allele regulates increased and decreased metabolites; P-value $<0.01$ 


\section{BIOINFORMATION \\ Discovery at the interface of physical and hiological sciencess}

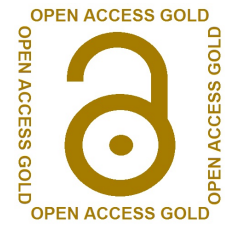

Association between rs823093 variant and metabolites

Sixteen metabolites showed remarkable associations with rs823093 variant at $\mathrm{P}<0.01$ such as Glycerol Isobutyrylcarnitine, Pantothenate, Lactate, 4-acetamidobutanoate, LDL, Serotonin (5HT), Gamma-glutamylleucine, Phosphate, Cholesterol, Caprylate, Oleate, Heptanoate, 7-methylguanine, Glucose and Nacetylornithine as listed in Table 4.

Table 5: Expression analysis of genes including NUCKS1, regulated by rs823093 variant in Parkinson's disease dataset

\begin{tabular}{lllll}
\hline Probe ID & Gene & t-statistics & $\begin{array}{l}\text { Fold change } \\
(\log 2)\end{array}$ & P-value \\
\hline 239929_at & PM20D1 & 1.08838876 & 0.37 & $2.87 \mathrm{E}-01$ \\
218700_s_at & RAB7L1 & 2.19856388 & 0.467 & $3.72 \mathrm{E}-02$ \\
218699_at & RAB7L1 & 2.12573885 & 0.397 & $4.34 \mathrm{E}-02$ \\
243777_at & RAB7L1 & -1.36547912 & -0.572 & $1.84 \mathrm{E}-01$ \\
226880_at & NUCKS1 & -4.18256211 & -0.861 & $2.99 \mathrm{E}-04$ \\
223661_at & NUCKS1 & -2.09624443 & -0.654 & $4.61 \mathrm{E}-02$ \\
224582_s_at & NUCKS1 & -1.72103478 & -0.316 & $9.74 \mathrm{E}-02$ \\
224581_s_at & NUCKS1 & -1.2063773 & -0.178 & $2.39 \mathrm{E}-01$ \\
217802_s_at & NUCKS1 & -1.03533831 & -0.183 & $3.10 \mathrm{E}-01$ \\
222424_s_at & NUCKS1 & -0.775199 & -0.142 & $4.45 \mathrm{E}-01$ \\
229353_s_at & NUCKS1 & -0.0254664 & -0.567 & $9.80 \mathrm{E}-01$ \\
225570_at & SLC41A1 & -1.88066702 & -0.25 & $7.15 \mathrm{E}-02$ \\
206910_x_at & CFHR2 & 1.40070154 & 0.268 & $1.73 \mathrm{E}-01$ \\
205913_at & PLIN1 & -0.49919732 & -0.287 & $6.22 \mathrm{E}-01$ \\
1558533_at & KRBA2 & 0.71072732 & 0.187 & $4.84 \mathrm{E}-01$ \\
206695_x_at & ZNF43 & -1.79511489 & -0.289 & $8.45 \mathrm{E}-02$ \\
222136_x_at & ZNF43 & -0.67058716 & -0.111 & $5.09 \mathrm{E}-01$ \\
201518_at & CBX1 & 0.77565557 & 0.832 & $4.45 \mathrm{E}-01$ \\
213265_at & PGA4 & 0.22672692 & 0.965 & $8.22 \mathrm{E}-01$ \\
204819_at & FGD1 & 1.7057522 & 0.364 & $1.00 \mathrm{E}-01$ \\
213438_at & NFASC & -0.97811674 & -0.189 & $3.37 \mathrm{E}-01$ \\
243645_at & NFASC & -0.87218298 & -0.304 & $3.91 \mathrm{E}-01$ \\
1552463_at & SERPINB11 & 0.99260234 & 0.435 & $3.30 \mathrm{E}-01$ \\
210923_at & SLC1A7 & -0.72614289 & -0.288 & $4.74 \mathrm{E}-01$ \\
225536_at & TMEM54 & -0.86172346 & -0.163 & $3.97 \mathrm{E}-01$ \\
209479_at & CCDC28A & -1.74253691 & -0.345 & $9.35 \mathrm{E}-02$ \\
228696_at & SLC45A3 & 0.5812718 & 0.124 & $5.66 \mathrm{E}-01$ \\
242372_s_at & MFSD4 & -1.11253201 & -0.808 & $2.76 \mathrm{E}-01$ \\
238862_at & MFSD4 & -0.43285076 & -0.188 & $6.69 \mathrm{E}-01$ \\
\hline
\end{tabular}

Validation of NUCKS1 gene expression in Parkinson's disease The expression of NUCKS1 gene in Parkinson's disease has been evaluated using GEO2R tool. Seven probes i.e. 226880_at, 223661_at, 229353_s_at, 224582_s_at, 217802_s_at, 224581_s_at and 222424_s_at were identified in the expression of NUCKS1 gene in gene expression profile of substantianigra of postmortem brain from Parkinson's disease patients. Each probe represent different region of NUCKS1 gene and may have same or different transcript. All these probes are found to be deregulated in Parkinson's cases compared with healthy individuals. Among these 226880_at is significantly deregulated with $\mathrm{P}=2.99 \mathrm{e}-04$ and $\log 2$ (fold change) $=$ -0.861 (Table 5). There are 18 different genes, which are regulated by rs823093 as described in Table 3. Simultaneously, evaluation of expression of other 17 genes with NUCKS1 revealed that three of them are also have different expression in Parkinson's cases i.e. RAB7L1, NFASC and MFSD4 at $\mathrm{P}<0.01$ and multiple testing correction (MTC) threshold 0.000345 (Table 5). Further, network analysis of NUCKS1 gene with other expressed genes regulated by rs823093 variant, revealed that NUCKS1 is co-expressed with ZNF43 and PLIN1genes and ZNF43 shared a protein domain with KRBA2 gene (Figure 2).

\section{Discussion:}

Genome-wide association studies have shown that PARK16 locus has significant association with Parkinson's disease [26, 27]. NUCKS1 is also reported as one of the important gene at PARK16 locus and has noteworthy association with PD [16]. NUCKS1 encodes a nuclear protein including phosphorylation sites for casein kinase 2 and cyclin-dependent kinases substrate. It is vertebrate specific gene ubiquities in the brain and peripheral tissues [29]. The casein kinase 2 has been reported to be involved in altering the dopamine signaling as well as hyper phosphorylation of alpha-synuclein [30,31] and cyclin-dependent kinases, suppress dopamine D1 signaling in the striatum by phosphorylation of postsynaptic protein DARPP-32 [32]. Some previous studies have also reported that cell-cycle protein mediates the degeneration of dopaminergic neurons [7, 33]. An earlier study reported that rs823128 variant of NUCKS1 might affect PD risk by altering the transcription factor-binding capability of the genes [34] and also reported as hub gene in a gene network analysis study on Parkinson's disease [35].

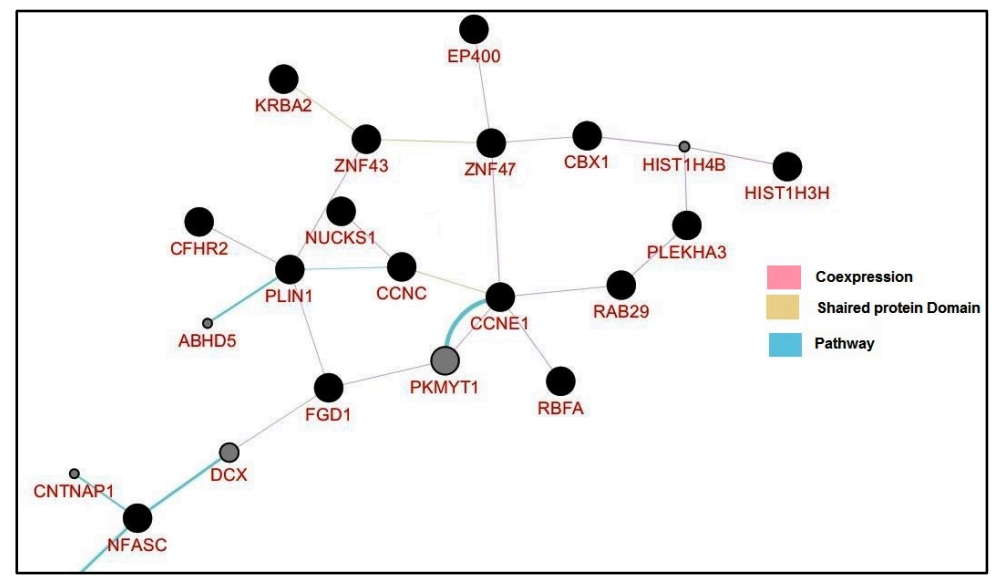

Figure 2: Correlation between NUCKS1 with other expressed genes

Hence, NUCKS1 may be crucial for cell cycle progression. Though a definite mechanism of NUCKS1 in PD is not known, it may 

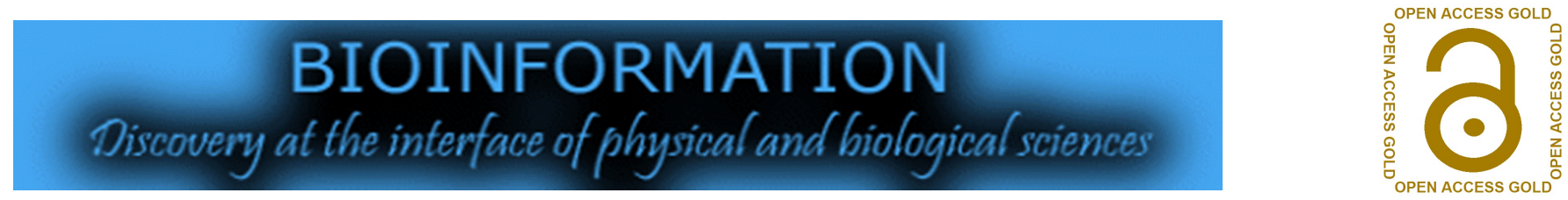

presumably be involved in the pathogenesis of PD. A complete functional analysis of rs823093 variant of NUCKS1 gene predicated a score of 6, on application of RegulomeDB, using PWM method, suggesting that rs823093 is likely to affect OTX2 motif (chr1: 205689224 - 205689231b) and is associated with histone modification in blood and strong transcription in various parts of brain besides, heart, kidney and liver. Application of HaploReg (version 4.1), also suggested that rs823093 is associated with enhancement of histone modification in blood verifying the findings in HaploReg (version 4.1). PhenoScanner GWAS analysis, showed that rs823093 is not only associated with Parkinson disease, but also is significantly associated with other diseases or phenotypes including Plateletcrit, Mucinous ovarian cancer, Chronic kidney disease, ovarian cancer, Ulcerative colitis, Late onset Alzheimers disease and many other. A study on Alzheimer's disease in Han chinese population also suggested that Parkinson's disease GWAS-Linked loci i.e. RAB7L1-NUCKS1 is associated with late Alzheimer's disease [36]. The rs823114 variant of NUCKS1 also indicates decreased risk of susceptibility to PD in Han Chinese male and association of three candidate genetic variants in RAB7L1/NUCKS1, MCCC1 and STK39 with sporadic Parkinson's disease [37, 38]. It is interesting that GA haplotype is reported as risk factor for PD and phenoscanner testify that rs823093 is associated with GA haplotype.

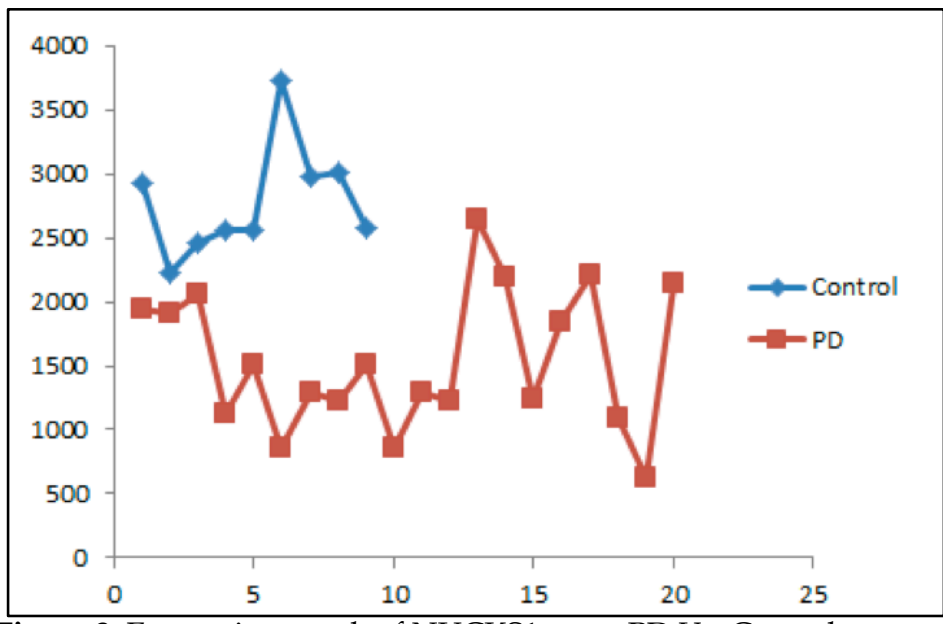

Figure 3: Expression graph of NUCKS1 gene: PD Vs. Control

PhenoScanner gene expression analysis showed that rs823093 is significantly associated with expression of multiple genes in multiple human tissues together with NUCKS1. Also rs823093 was identified to be expressively associated with other 16 metabolites including Serotonin (5HT) using PhenoScanner metabolites option.
Interestingly serotogenic dysfunction has a direct relevance to Parkinson's disease non-motor symptoms, like depression, fatigue, weight changes, and visual hallucinations. Substatianigra of postmortem human brain exhibited different expression of NUCKS1 gene in PD patients as compared with healthy samples $(\mathrm{PD}=16$; Healthy $=09)$, likewise the gene expression graph (Figure 3) for 226880_at probe depicts that NUCKS1 is down regulated in PD patients. Additionally, NUCKS1 is co-expressed with ZNF43 and PLIN1 genes where ZNF43 share a protein domain with KRBA2, in network analysis. Therefore it is presume that these three genes may also works as susceptible gene for PD pathogenesis but more study has to be needed.

\section{Conclusion:}

NUCKS1 is reported as one of the significant gene at PARK16 locus and has remarkable connotation with PD but its mechanism is not yet known. In current study a comprehensive functional analysis of rs823093 variant of NUCKS1 gene has been done using gene expression, disease association, network and metabolite analysis. The findings of stated analysis verified the possible association of NUCKS1 gene with PD, which may serve as susceptibility marker for PD.

\section{Acknowledgement:}

We wish to thanks to National Academy of Sciences India (NASI), Allahabad (Grant No. NAS/508/2/2016-17) for the financial support as NASI Senior Scientist Platinum Jubilee Fellowship and are also thankful to Biotech Park, Lucknow for providing workspace. We also express sincere thanks to Prof. Krishna Mishra, Professor, III-T Allahabad for her valuable comments on the manuscript.

\section{References:}

[1] Picillo M et al. Journal of Parkinsonism and Restless Legs Syndrome 2016 6:1[DOI: 10.2147/JPRLS.S85488].

[2] Das SK et al. Neurology 2010 75:1362 [PMID: 20938028].

[3] Miller DB \& O'Callaghan JP, Metabolism 2015 64:S40 [PMID: 25510818].

[4] He T et al. Neurosci. Lett 2017 657:179 [PMID: 28807727].

[5] Delamarre A \& Meissner WG, Presse Med 2017 46:175[PMID: 28189372].

[6] Simon-Sanchez J et al. Nature Genetics 2009 41:1308[PMID: 19915575].

[7] Grundt K et al. Biochem. Biophys. Res. Commun 2004 323:796[PMID: 15381070].

[8] Huang P et al. Biomed Res Int 20187969068 [PMID: 29619377].

[9] Xu et al. Spine 2017 42:1629 [PMID: 28338576]. 


\section{BIOINFORMATION \\ Discovery at the interface of physical and biological sciences}

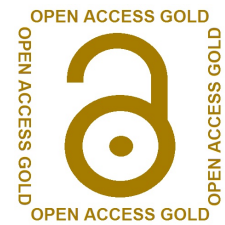

[10] Giunti et al. Mol Clin Oncol. 2019 10:331 [PMID: 30847170].

[11] Yuan et al. J Cell Physiol 2019 [PMID: 30710349].

[12] Huang et al. Carcinogenesis 2019 40:370 [PMID: 30371738].

[13] Dunlop MH et al. The Journal of Biological Chemistry 2012 287: 12343 [PMID: 22375013].

[14] Nalls MA et al. Nature Genetics 2014 46:989 [PMID: 25064009].

[15] Satake W et al. Nature Genetics 2009 41:1303 [PMID: 19915576].

[16] Boyle AP et al. Genome Res 2012 22:1790 [PMID: 22955989].

[17] Ward LD \& Kellis M, Nucleic Acids Res 2012 40:D930 [PMID: 22064851].

[18] Ward LD \& Kellis M, 2016 44, D877 [PMID: 26657631].

[19] Staley JR et al. Bioinformatics 2016 32:3207 [PMID: 27318201].

[20] Shin SY N et at. Genet 2014 46:543 [PMID: 24816252].

[21] Kettunen J et al. Nat. Commun 2016 7:11122 [PMID: 27005778].

[22] Lesnick TG et al. 2007 PLoS Genet 3:e98.[PMID: 17571925].

[23] Barrett T et al. Nucleic Acids Res 2012 41:D991 [PMID: 23193258].

[24] Warde-Farley D, Nucleic Acids Res 2010 38:W214.[PMID: 20576703].
[25] Tucci A et al. Eur. J. Hum. Genet 2010 18:1356 [PMID: 20683486].

[26] Pihlstrom L et al. J. Hum. Genet 201560 :357[PMID: 25855069].

[27] Ostvold AC et al. Eur. J. Biochem 2001 268:2430 [PMID: 11298763].

[28] Ishii A et al. FEBS Lett 2007 581:4711[PMID: 17868672].

[29] Rebholz H et al. Biol. Psychiatry 2013 74:113 [PMID: 23290496].

[30] Peng J et al. Scientific Reports 2019 9:1[PMID: 30886221].

[31] Hoglinger GU et al. Proc. Natl. Acad. Sci. U.S.A. 2007 104: 3585 [PMID: 17360686].

[32] Bai Y et al. Neuroreport 2017 28:936 [PMID: 28749816].

[33] Chen JA et al. Neurodegener Dis 2018 18:191 [PMID: 30089309].

[34] Xi-Chen et al. Molecular Neurobiology 2017 54:308 [PMID: 26738859]

[35] Zhu W et al. Genes Genet Syst 2018 93:59[PMID: 29607885].

[36] Wang L et al. J Neural Transm 2016 123:425 [PMID: 26914237].

[37] Xia H et al. Genet. Mol. Res 2015 14:2978[PMID: 25966061].

[38] Politis M \& Niccolini F, Behavioral Brain Research 2015 277:136 [PMID: 25086269]. 


\section{BIOINFORMATION}

Discovery at the interface of physical and biological sciences
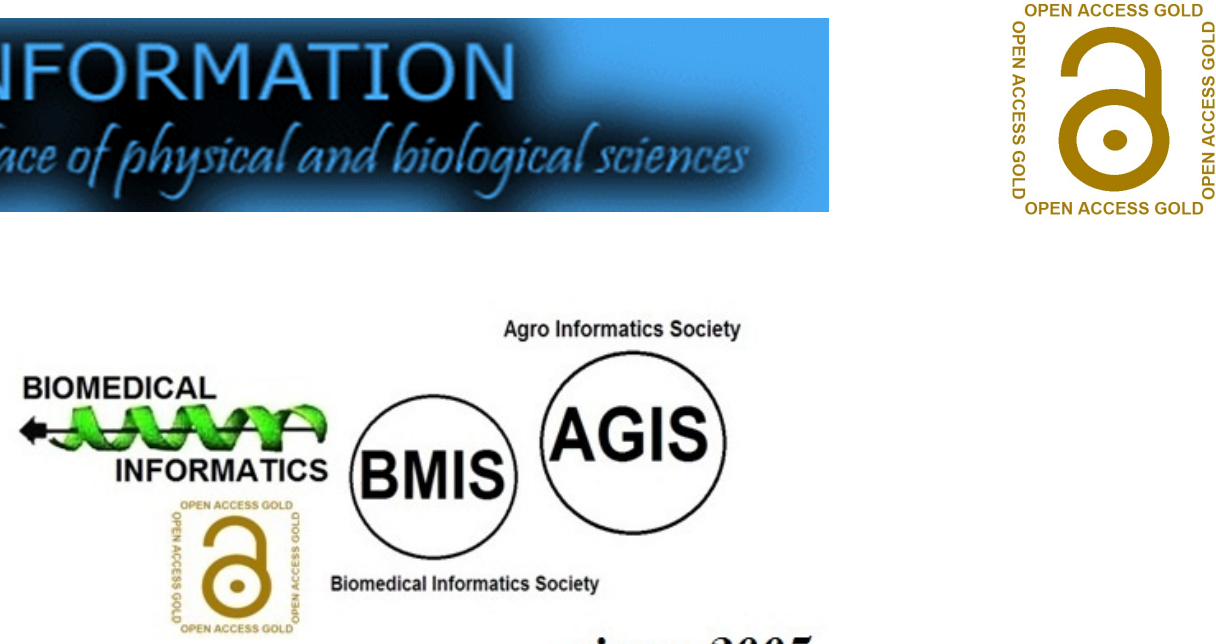

since 2005

\section{BIOINFORMATION}

Discovery at the interface of physical and biological sciences

\section{indexed in}

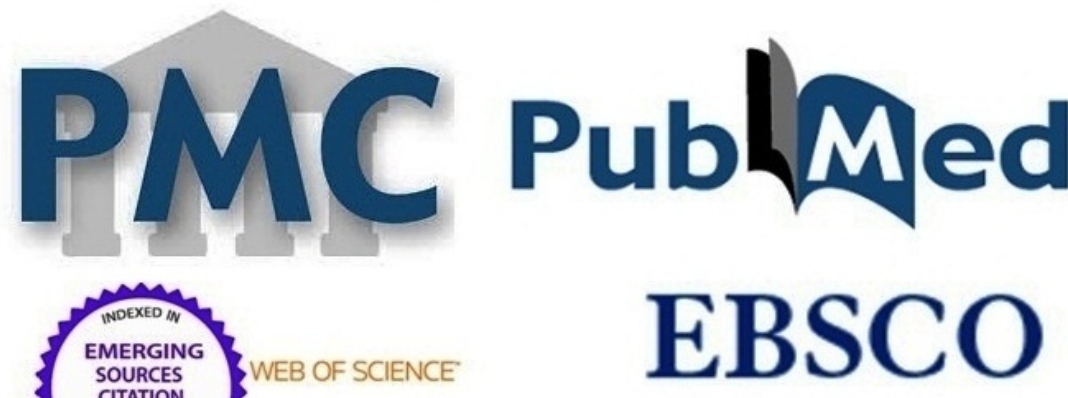

Web of

Science

Group

4 Crossref
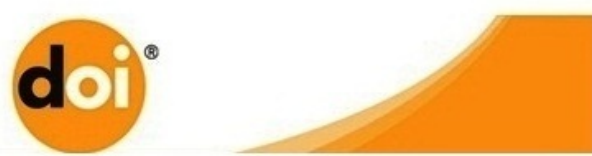

ResearchGate

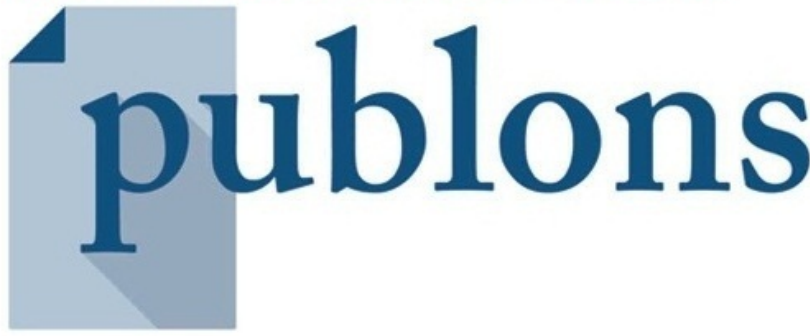

$\mathbf{G}$ 